\title{
QUALE «DEMOCRAZIA COSTITUZIONALE»? LA CORTE FEDERALE NELLA POLITICA TEDESCA E IL PROBLEMA DELLA COSTITUZIONE EUROPEA
}

\author{
di Gian Enrico Rusconi
}

\section{La Corte costituzionale nella politica tedesca}

$\mathrm{La}$ Germania offre una interessante versione contemporanea dello «Stato costituzionale» o della «democrazia costituzionale» con le sue tipiche tensioni tra responsabilità politica parlamentare e giustizia costituzionale. Un esempio tanto più istruttivo in quanto si pone all'incrocio tra la tradizione tedesca dello «Stato di diritto» e la tendenza ad un «nuovo costituzionalismo» che suscita crescente attenzione nelle scienze politiche e giuridiche ${ }^{1}$.

Ma c'è di più. La centralità del ruolo della Germania in Europa fa sì che questa problematica si proietti sulla costruzione politico-costituzionale dell'Unione europea. L'istituzionalizzazione dell'Europa politica e la semplice ipotesi di una Costituzione europea rimettono in gioco i concetti classici di popolo, sovranità, statualità e legittimazione democratica e aggiornano i dilemmi del costituzionalismo e della Costituzione come statuto

1 Tra i lavori collettanei apparsi in paesi e contesti culturali diversi, ma con problematiche convergenti, segnalo Elkin e Soltan (1993), Preuss (1994b), Zagrebelsky, Portinaro e Luther (1996), Bellamy e Castiglione (1996a). Inutile dire che questa problematica è già presente nel dibattito precedente sul costituzionalismo. In merito ricordo due autorevoli studiosi italiani: Giovanni Sartori e Nicola Matteucci. Da Sartori $(1987,27)$ tolgo l'avvertimento: «Quando un problema politico - e il costituzionalismo è inevitabilmente soluzione giuridica di un problema politico - è depoliticizzato, le conseguenze effettive di un atteggiamento giuridicamente "neutrale" sono e restano per quanto involontariamente politiche; e tali a tutto vantaggio dei demagoghi e dei despoti». E Matteucci constata che «il costituzionalismo è oggi affidato soprattutto alla giurisprudenza delle Corti costituzionali che attuano appunto la giustizia costituzionale», precisando: «La Corte deve interpretare la norma costituzionale tenendo presente la qualità dei tem$\mathrm{pi}$, conciliare il principio di legalità con quello di equità e argomentare razionalmente la propria sentenza. Proprio come il giudice di common law che cerca il precedente più adeguato al caso. Insomma il suo procedere è (o dovrebbe essere) del tutto simile a un law finding e non a un law making» $(1993,168)$. Segnalo infine il saggio di Guarnieri e Pederzoli (1997) e, sul nesso tra Costituzione e federalismo, Pizzetti (1996).

RIVISTA ITALIANA DI SCIENZA POLITICA / a. XXVII, n. 2, agosto 1997 
delle libertà, come contratto politico e come forma di governo. In questa ottica il progetto europeo acquista i tratti di una democrazia o Stato costituzionale tutto da esplorare, per il quale il modello tedesco diventa molto istruttivo.

Cominciamo da un interrogativo che può apparire improprio o semplicistico ma che ha una sua forza di provocazione: chi «decide» in Germania? Se rivolgessimo questa domanda ad un pubblico mediamente informato sulle cose tedesche, raccoglieremmo reazioni interessanti. È facile prevedere che la grande maggioranza delle risposte attribuirebbe il «chi decide in Germania» al cancelliere oppure, in alternativa frequente in questi ultimi tempi, alla Bundesbank. Ma in questo secondo caso la risposta avrebbe anche il tono polemico della denuncia di una usurpazione del potere decisionale da parte della banca centrale. Nessuno o quasi degli interpellati invece nominerebbe la Corte costituzionale federale (il Bundesverfassungsgericht) come «decisore» politico in Germania. Anzi si stupirebbe se altri lo facesse.

Che la Corte federale attraverso il controllo di costituzionalità della attività legislativa sia in Germania il decisore ultimo di estrema rilevanza politica - di rilevanza non contingente ma sistematica - è una convinzione che si forma soltanto osservando con continuità la sua attività e l'effetto dei suoi pronunciamenti sul sistema tedesco nel medio e lungo periodo. Del resto il peso politico e psicologico di cui gode la Bundesbank in Germania dipende in non piccola parte dal fatto che la Corte federale considera imperativo costituzionale la «sicurezza della stabilità dei prezzi» che è assegnata come scopo primario alla Banca stessa e in futuro alla Banca centrale europea ${ }^{2}$.

$\mathrm{Ma}$ l'attività del Bundesverfassunsgericht e la sua influenza sulla politica tedesca sono tutt'altro che esenti da critiche e da proteste da parte dell'opinione pubblica, degli uomini politici e degli stessi studiosi. In questi ultimi anni le controversie hanno raggiunto un'intensità e una eco pubblica tale da far dire ad un noto studioso e giudice costituzionale, Ernst-Wolfgang Böcken-

2 La nuova versione dell'art. 88 del Grundgesetz recita: «Il Bund istituisce una banca valutaria e di emissione, come Banca federale. Le sue funzioni e competenze possono essere trasferite, nel quadro dell'Unione europea, alla Banca Centrale Europea, che è indipendente ed è vincolata allo scopo primario della sicurezza della stabilità dei prezzi». 
förde, che la Corte federale è «minacciata dal collasso». Collasso imputabile apparentemente al sovraccarico di lavoro indotto dall'attivismo dei giudici, ma nella sostanza alla natura delle tensioni da esso prodotte che mettono in crisi l'indispensabile rapporto di fiducia su cui si fonda l'esercizio della giustizia costituzionale ${ }^{3}$. Anche un altro noto giurista, Erhard Denninger, davanti alla estensione delle proteste pubbliche contro l'operato della Corte, ha recentemente parlato di «grave incertezza del diritto» in Germania ${ }^{4}$.

Ricordo qui alcune delle sentenze più significative della Corte di questi ultimi anni: l'approvazione dell'impiego di truppe tedesche out of area Nato, con l'abbandono della dottrina e della prassi del rigoroso astensionismo militare tedesco che durava dal 1945; la reinterpretazione restrittiva dell'art. 16 della Costituzione riguardante il diritto d'asilo, che pone così termine ad una prassi molto liberale della Germania in questo ambito; la sentenza sulla liceità dei blocchi stradali nel corso di dimostrazioni di protesta e quella sul carattere non diffamatorio dell'affermazione (che è nel contempo una citazione di Kurt Tucholsky) «i soldati sono assassini». Particolarmente significativa, per l'intensità delle contestazioni sollevate, è la sentenza sui criteri che devono regolare la presenza del crocefisso nelle aule delle scuole pubbliche.

Ma la sentenza cui dovremo prestare particolare attenzione è quella sul trattato di Maastricht, nella quale la Corte costituzionale parla di limiti di competenza delle istituzioni europee di fronte ai diritti di sovranità del Parlamento tedesco. È una sentenza che ha fatto parlare di «sorveglianza» della Corte federale sull'Unione europea .

Vorrei tuttavia aggiungere che tra le conferme del ruolo cruciale della Corte nella vita politica tedesca va annoverato paradossalmente - il suo non-intervento nel corso dell'evento più importante per la Germania di questi anni, la riunificazione.

3 «In nessun organo dello Stato costituzionale democratico viene investita tanta fiducia come nella Corte costituzionale. Infatti è sempre aperto l'interrogativo in che misura $\mathrm{i}$ giudici, chiamati a garantire la Costituzione, non ne diventino nel contempo " $\mathrm{i}$ padroni"». Dem Bundesverfassungsgericht droht der Kollaps, in «Frankfurter Allgemeine Zeitung», 24 maggio 1996: si tratta del discorso tenuto da Böckenförde in occasione della fine del suo mandato di giudice costituzionale, ricoperto dal 20 dicembre 1983 al 3 maggio 1996.

4 Denninger (1995, 425-450).

5 Tomuschat (1993). 
Oltre 16 milioni di abitanti di quello che sino al 1989/90 era lo Stato internazionalmente riconosciuto della Repubblica democratica tedesca (Ddr), hanno potuto «aderire» o «entrare» nella preesistente Bundesrepublik occidentale ai sensi del Grundgesetz, la Legge fondamentale che era stata concepita come provvisoria proprio in attesa della riunificazione del popolo tedesco. In termini formali costituzionali la riunificazione tedesca è avvenuta di fatto con l'applicazione dell'art. 23 che prevedeva la semplice «entrata» dei Länder orientali nella Bundesrepublik ${ }^{6}$. Anziché predisporre il passaggio verso «una nuova Costituzione adottata dal popolo tedesco con libera decisione» ai sensi dell'art 146, la decisione di applicare l'art. 23 equivaleva al riconoscimento del Grundgesetz come la Costituzione definitiva per l'intero popolo tedesco.

$\mathrm{Al}$ di là della fondatezza di questa interpretazione, quanto è accaduto è la dimostrazione che la costruzione costituzionale intrapresa per decenni dalla giurisprudenza della Corte, sulla base del testo originario del Grundgesetz, ha raggiunto una solidità e una flessibilità eccezionali. Non è semplicemente la conferma della bontà di quella che nel 1949 era stata pensata come una Costituzione a tempo, in attesa di un testo definitivo per l'intera Germania unita, ma è il coronamento di una attiva «politica della Costituzione» che nel 1989/92 ha superato la sua prova ultima. In questi anni infatti viene esclusa la necessità costituzionale di procedere ad una consultazione popolare diretta. La «libera decisione» del popolo tedesco unito, evocata nel Preambolo del Grundgesetz, viene surrogata dal Trattato di unificazione stipulato nel 1990 tra i due governi della Brd e della (ancora esistente) Ddr.

Ebbene, in questa catena di eventi e decisioni politiche la Corte federale non interviene mai. Dal punto di vista formale la sua passività è ineccepibile dal momento che non è mai stata chiamata a pronunciarsi. Oltretutto nel periodo che stiamo considerando le opinioni dei costituzionalisti tedeschi sono spaccate in due tra i fautori della semplice «entrata» dei Länder orientali nella Bundesrepublik e quelli che invece auspicano un pro-

6 L'art. 23 (nella vecchia versione ora integralmente sostituita) prevedeva che il Grundgesetz entrasse in funzione in «altre parti della Germania dopo la loro entrata (Beitritt)». L'art. 146 (vecchia versione) diceva: «questo Grundgesetz perde la sua validità il giorno in cui entra in vigore una Costituzione (Verfassung) che è stata adottata con libera decisione dal popolo tedesco». 
cedimento (referendario) di approvazione di una nuova Costituzione sia pure ricalcata sul Grundgesetz. In verità, il Parlamento tedesco istituisce una Commissione ad boc per la messa a punto di alcune modifiche e innovazioni costituzionali, sulle quali si sarebbe dovuta eventualmente pronunciare la Corte. Ma il risultato di questa Commissione è un nulla di fatto. È lecito chiedersi se all'incapacità innovativa di questo organo politico parlamentare («bicamerale»), segnato pesantemente dalla logica degli schieramenti partitici, non sia estraneo un certo scetticismo aprioristico nei suoi riguardi in contrasto con l'enorme prestigio che la Corte costituzionale ha accumulato in decenni di giurisprudenza. Così gli anni cruciali 1989/92 confermano che in Germania la Costituzione e i suoi eventuali emendamenti sono affidati alla Corte federale, come sua riserva esclusiva. Questo affidamento è onorato dalla Corte con l'emissione di una serie di sentenze (alcune delle quali ricordate sopra) di grande rilievo e fortemente controverse che hanno riacutizzato la tensione tra le competenze della decisione politica e quella della giurisdizione costituzionale (Verfassungsgerichtsbarkeit).

È bene sottolineare che questo tipo di tensione non è imputabile specificatamente al sistema tedesco: basti ricordare le frizioni e i contrasti provocati dalle iniziative della Corte suprema degli Stati Uniti. Il caso tedesco tuttavia offre un interesse del tutto particolare perché si muove nella tradizione dello «Stato di diritto» euro-continentale e si proietta sulla costruzione politica dell'Unione europea. Non a caso, nella sua sentenza sul trattato di Maastricht la Corte di Karlsruhe ha rivendicato per sé la competenza di «verificare se atti giuridici delle istituzioni e degli organi europei rimangono nei limiti dei diritti di sovranità loro assegnati o li travalicano». Contestualmente la Corte ha fatto una serie di affermazioni sulla natura politica dell'Unione europea tanto impegnative da poter essere lette come una sorta di ipoteca sullo sviluppo politico dell'Europa.

\section{Che cosa è la democrazia costituzionale}

Per qualificare il sistema politico-istituzionale della Germania odierna gli studiosi del diritto usano spesso l'espressione «democrazia costituzionale» (Verfassungsdemokratie) ripresa magari dal dibattito americano ma riconiugata in un quadro concettuale e storico-politico assai differente. Tale espressione 
si affianca ad altre coniate in ambito politologico quali «democrazia del cancelliere» (Kanzlerdemokratie) e «democrazia della contrattazione» (Verbandlungsdemokratie).

Queste prospettive analitiche si intrecciano in modo peculiare. Tutte hanno in comune la democrazia: ma questo riferimento ha senso e contenuto molto diverso a seconda se esso segnala la «sovranità della Costituzione» affidata ai suoi interpreti autorizzati; se pone l'accento su una formula di governo (il cancellierato) nel quale culmina un particolare sistema politico democratico; oppure se il principio democratico è individuato nella pratica della contrattazione e del bilanciamento delle competenze, non solo in ottemperanza al principio federale ma anche secondo lo schema neo-corporativo o consociativo che è caratteristico del sistema tedesco ${ }^{7}$. Prese insieme, le tre ottiche disegnano l'architettura politico-istituzionale tedesca che nei suoi processi di legittimazione, rappresentanza e decisione è assai più complessa di quanto non appaia da certe immagini correnti che la descrivono come un ordine compatto e lineare.

L'aggettivo «costituzionale» accostato qui alla democrazia non indica una qualche variante nella struttura del sistema politico rappresentativo e esecutivo, ma mette a fuoco il principio generale delle garanzie democratiche. Questo esige il controllo di legittimità delle azioni e dei comportamenti degli attori sia politici che sociali - controllo affidato alla Corte costituzionale che in questo modo interviene direttamente sui modi di funzionamento della democrazia. Ritroviamo così in altra forma gli elementi del costituzionalismo - statuto delle libertà (diritti fondamentali), forma di governo (democrazia del cancelliere), patto politico (democrazia della contrattazione). O meglio, siamo posti davanti all'esigenza di trovare un equilibrio tra di essi. $\mathrm{Da}$ questo equilibrio prende forma la democrazia costituzionale nella variante tedesca.

In particolare viene in primo piano la questione della sovranità e del suo esercizio concreto. La democrazia costituzionale

7 La letteratura sulla natura consociativa e strutturalmente compromissoria del sistema tedesco è vastissima. Da un punto di vista costituzionale si veda un saggio di Böckenförde scritto a metà degli anni settanta nel quadro del dibattito sulla «governabilità», ma tuttora valido (ora in Böckenförde 1991). L'autore, dopo aver parlato della «situazione costituzionale» tedesca «come di un compromesso di classe istituzionalizzato», aggiunge che «è ozioso chiedersi se questo compromesso esista solo di fatto, come dato politico, e se la Costituzione contenga una garanzia istituzionale per la sua persistenza». 
riconosce innanzitutto la «sovranità della Costituzione», di cui la Corte è insindacabile interprete. Se in una democrazia la sovranità è qualificata operativamente dall'istanza di decisione ultima, allora questa prerogativa spetta alla Corte. Questo modo di concepire e praticare la sovranità può entrare in contrasto con il principio della sovranità del popolo e/o del Parlamento.

Questa problematica, così come si manifesta nella esperienza tedesca, si lascia articolare in alcuni punti:

a) I confini di competenza tra Parlamento e Corte sono stabiliti dalla Costituzione stessa che in tema di legislazione assegna il primato al Parlamento, riservando tuttavia alla Corte l'ultima parola. Il delicato e controverso rapporto tra giurisdizione dei giudizi costituzionali e iniziativa dei politici porta a parlare di «giuridificazione della politica» e «politicizzazione della giurisprudenza costituzionale».

b) Alla Corte sono riconosciute competenze non solo di controllo passivo di costituzionalità ma di iniziativa attiva per «l'attuazione della Costituzione», intesa come «ordinamento oggettivo di valori». Di conseguenza è inevitabile che alcune decisioni della Corte appaiano politiche in senso forte ovvero che la sua giurisprudenza sia (o appaia) politicizzata quando entra in concorrenza e in qualche caso in contrasto con l'iniziativa legislativa o di altri attori politici.

c) Per la verità, la classe politica (tedesca) quando legifera tiene sempre in conto e anticipa le possibili reazioni della Corte; spesso i politici professionali anziché assumersi autonomamente $\mathrm{i}$ rischi della decisione politica, speculano sul possibile atteggiamento della Corte, deresponsabilizzandosi in anticipo presso i loro elettori. Questo atteggiamento porta a sovraccaricare l'iniziativa legislativa di argomenti e controargomenti legali. Quando il confronto politico degli interessi prende la forma di una interpretazione giuridico-costituzionale, allora la decisione si sposta dall'arena politico-parlamentare alla sala del tribunale. Ma la «giuridificazione della politica» non è imputabile solo all'attivismo dei giudici bensì anche al comportamento dei politici.

d) La Corte costituzionale è prigioniera del paradosso che si genera tra la politicità della sua azione e la apoliticità richiesta soggettivamente ai giudici nell'elaborazione del loro giudizio. L'insindacabilità della giurisdizione costituzionale è di fatto prerogativa di poche persone, scelte con criterio politico spartitorio, che decidono praticamente senza controllo (attraverso 
sentenze pronunciate spesso con il minimo scarto tra maggioranza e minoranza).

e) Che la scelta dei giudici costituzionali sia altrettanto importante, se non più importante delle elezioni parlamentari, non è un'aporia da trattare con disinvoltura. L'unico correttivo al virtuale abuso dell'insindacabilità dei giudici è il loro autocontrollo (judicial self-restraint / richterliche Zurückbaltung). Dubbia appare invece l'applicazione della cosiddetta political-question doctrine di ispirazione americana, che definisce a priori quali questioni siano da lasciare al legislatore o quali all'esecutivo; di fatto essa è già praticata dalla Corte, quando dichiara che determinate decisioni sono di competenza del governo o del Parlamento.

f) La Corte non ha alcun potere esecutivo e implementativo delle proprie sentenze. Il suo potere effettivo si fonda sulla disponibilità degli altri organi costituzionali (in prima linea del governo e del Parlamento) a seguire le sue indicazioni. Fiducia e prestigio sono le risorse insostituibili per la Corte. Quando non riesce ad argomentare in modo persuasivo, il suo pronunciamento appare una pretesa di potere impropria e un arbitrio che crea non solo insicurezza nel diritto ma sconcerto sui fondamenti della democrazia.

A dispetto della mole di questi problemi, non solo l'istituzione regge ma il suo ruolo è destinato a crescere a mano a mano che l'asse delle grandi decisioni si sposta dal piano nazionale a quello europeo. Il coinvolgimento della Corte di Karlsruhe nella questione europea è molto di più di un «dovere d'ufficio». L'evoluzione dello Stato legislativo parlamentare in Stato democratico giurisdizionale che si delinea in Germania, anche grazie alla interpretazione dei diritti fondamentali come norme di principio oggettivo affidate all'esclusiva competenza della Corte, diventa a livello europeo la messa in cantiere di uno Stato costituzionale di tipo nuovo.

\section{La Costituzione prende il posto dello Stato?}

Iniziamo da due approcci che hanno assunti di valore divergenti (liberal-conservatore il primo, radical-democratico il secondo) ma convergono nel criticare il ruolo politico della Costituzione o meglio l'uso politico che di essa fanno i suoi interpreti autorizzati (i giudici costituzionali). Possiamo anticipare che 
gli argomenti dei due approcci, basati su concezioni differenti di democrazia e di politica democratica (convenzionalmente classificabili di «destra» e di «sinistra») si neutralizzano a vicenda rivelandosi entrambi inconcludenti davanti alla realtà della «democrazia costituzionale» così come viene interpretata dalla Corte.

Josef Isensee sin dal titolo del suo saggio La Costituzione come patria. Sulla rimozione dello Stato da parte dei tedeschi (1989) esprime chiaramente un punto vista polemico piuttosto influente negli ambienti più conservatori della giurisprudenza tedesca ${ }^{8}$. La sua critica alla «Costituzione come patria» prende le mosse da lontano, dalla irrisolta questione della identità dei tedeschi. Dopo la catastrofe del Terzo Reich e di fronte alla realtà apparentemente insuperabile della divisione della nazione, la cultura politica tedesca del dopoguerra ha ripreso la vecchia idea che il luogo della vera identità tedesca sia la Bildung, la Kultur, la Innerlichkeit. Ma nella Repubblica federale la Bildung come fattore positivo di identificazione diventa poco alla volta il Grundgesetz, la Legge fondamentale. Si tratta di una «soluzione luterana», perché ci si muove «senza tradizione e senza istituzione e si costruisce sulla pura parola scritta. Sola scriptura: das Bonner Grundgesetz. Questo costituisce il simbolo, forse l'unico, per il consenso positivo di fondo in una situazione di dissenso pluralistico. Diventa il grande tabù che ognuno rispetta» (ibidem, 14) .

Questo punto di vista polemico presuppone un giudizio disincantato sulla stessa Legge fondamentale e sulla sua genesi. Stesa sotto lo stretto controllo delle potenze occupanti - scrive Isensee - la Costituzione provvisoria di Bonn è priva del requisito fondamentale di una Costituzione democratica: quello di essere espressione di un libero popolo sovrano. Invece (come già aveva detto anni prima Ernst Forsthoff) «la Legge fondamentale non è il risultato di una decisione politica ma il prodotto di una situazione, più esattamente di una condizione di debolezza senza precedenti quale conseguenza della guerra perdu-

8 Ricordo che Josef Isensee insieme a Paul Kirchhof è il curatore dell'autorevole Handbuch des Staatsrechts der Bundesrepublik Deutschland, giunto al X volume.

9 La polemica di Isensee è evidentemente contro le tesi del «patriottismo costituzionale», anche se non cita né Sternberger né Habermas. Sulla rilevanza del concetto di Verfassungspatriotismus nel dibattito tedesco ed europeo rimando a Rusconi (1993; 1997). 
ta». Questo dato di fatto, per la verità, non ha impedito che la Legge fondamentale fosse accettata e godesse di un consenso eccezionale («un plebiscito continuo») trasferito alla stessa Corte costituzionale. Ma le ragioni di questo successo non risiedono nell'istituzione bensì nella prosecuzione inerziale, per così dire, di vecchie virtù tradizionali tedesche (senso dello Stato, etica del lavoro, ecc.). Detto questo, il Grundgesetz si rivela una buona Costituzione per la sobrietà ed essenzialità delle sue formulazioni: ad esse manca il pathos civil-religioso tipico di molte Costituzioni democratiche. $\grave{E}$ in questo spirito che $i$ padri costituzionali hanno deciso che in caso di conflitto attorno alle norme l'ultima parola spettasse in modo insindacabile al giudice, unico garante della Costituzione.

Su questo punto - osserva Isensee - devo modificare la tesi luterana del solo affidamento alla «parola» costituzionale con un momento cattolico: nella controversia sulla vera parola c'è una istanza ultima che decide in modo vincolante. Il conflitto costituzionale si rivela come vero e proprio conflitto legale. La qualità giuridica, che è propria della Legge fondamentale, si rivela come causa essenziale del suo successo $(1989,16)$.

$\mathrm{Ma}$ con il passare del tempo la sobrietà originaria del testo costituzionale è stata compensata, ipercompensata da sempre nuovi costrutti giuridici. «I diritti fondamentali concepiti alla maniera liberale acquistano sempre nuove dimensioni al di là dei diritti di libertà del singolo: diventano valori oggettivi e norme fondamentali decisivi in termini di valore». Nella Costituzione vengono ritrovati programmi politici, desiderata morali, filantropici o modelli culturali. La Costituzione interpretata come un insieme di valori oggettivi diventa fonte di sempre nuovi diritti soggettivi. Di conseguenza la discussione politica si trasfor$\mathrm{ma}$ in esegesi costituzionale e viceversa.

Questa situazione è ambivalente: da un lato la politica perde la sua distruttiva litigiosità, diventa prevedibile, ma dall'altro evita ogni rischio decisionale. Inizia il processo di giuridificazione della politica (Juridifizierung der Politik). Parallelamente «si compie una metamorfosi della Costituzione: da legge suprema si trasforma in programma di integrazione politica, da ordinamento statuale tematicamente limitato si trasforma in Costituzione totale, illimitata, virtualmente onnicompetente per la società e lo Stato». Reciprocamente, «la politica cacciata dal diritto ritorna sotto altra veste come interpretazione politica del diritto (...). La politica costituzionale deve mettere in atto, otti- 
mizzare e persino ampliare il diritto costituzionale nelle nuove condizioni del tempo, adattare la legge alla Costituzione e la Costituzione alla realtà. In questo gioco dialettico è difficile dire chi alla fine tiene in mano le fila, se il politico o il giurista» $(1989,22)$. Tutto questo - secondo Isensee - porta alla sostituzione della Costituzione allo Stato, porta alla contrapposizione della Costituzione allo Stato. La classica «ragion di Stato» viene sostituita dalla «ragione costituzionale» e la Costituzione diventa «la patria».

Mi limito ad un solo commento: Isensee, tutto preso dalla sua polemica ( $\ll$ non ci sono più cittadini tedeschi, ma cittadini della Costituzione»), non sospetta che la trasformazione da lui stigmatizzata possa segnalare non già la negazione dello Stato ma la sua democratizzazione. Il suo sarcasmo nei confronti del «zelotismo costituzionale», del «moralismo o religiosità costituzionale» di coloro che fanno della dichiarazione dei diritti umani un surrogato secolare della Bibbia, rischia di andare oltre il segno. Soprattutto non si capisce quale correttivo politico sia necessario per evitare una trasfigurazione pseudoreligiosa della Costituzione.

\section{Sovranità della Costituzione vs. sovranità popolare}

La giuridificazione della politica è denunciata anche, dal punto di vista radical-democratico, da una filosofa e da una politologa che ragionano sul concetto democratico di sovranità. Cominciamo da Volkssouveränität versus Konstitutionalismus di Ingeborg Maus, una delle più note e decise critiche del giurisdizionalismo costituzionale tedesco, considerato corpo estraneo al principio democratico ${ }^{10}$.

La differenza tra il tipo di Costituzione della «democrazia» e quello del «costituzionalismo» non sta nell'asserito primato delle libertà individuali - leggiamo - ma nelle modalità della sua attuazione. Nella Costituzione «costituzionalista» la difesa istituzionale delle libertà si fonda sulla concorrenza tra gli apparati statali che si dividono la sovranità. Nel principio democratico invece la «indivisibilità della sovranità popolare» consente una articolazione solo funzionale dei poteri: la sostanza della 
sovranità sta nella pienezza del potere legislativo: né l'esecutivo né il giudicativo possono partecipare alla legislazione. I modelli del costituzionalismo trattano il potere politico come problema primario e solo secondariamente lo limitano tramite i diritti di libertà e il bilanciamento dei poteri: la libertà è definita essenzialmente in modo negativo. Invece nel modello della sovranità popolare esiste un nesso molto stretto tra i diritti di libertà metapositivi e la partecipazione politica ${ }^{11}$. L'unione personale democratica del bourgeois e del citoyen, lo status activus della cittadinanza è garantito soltanto in una Costituzione che non sia mera costituzionalizzazione del potere e nella quale la volontà dei cittadini non venga sostituita dal potere degli esegeti costituzionali.

Sullo sfondo di questa filosofia politica anche Heidrun Abromeit (1995) contrappone la «sovranità popolare» alla «sovranità della Costituzione», dando alla sovranità un significato politologico specifico di competenza o diritto di un organo dello Stato alla decisione finale ovvero di competenza o diritto di approvare, come istanza definitiva, un'azione intrapresa da altri organi. Segue una tipologia a seconda che il detentore di tale sovranità sia il popolo, il Parlamento o la Costituzione. A questi tipi corrispondono rispettivamente tre diversi sistemi politici storici: lo svizzero, l'inglese e il tedesco. Non intendo in questa sede verificare la significatività di questa tipologia ${ }^{12}$. Mi limito a registrare che in questo confronto a tre il sistema tedesco viene presentato in termini fortemente critici perché la «sovranità della Costituzione» in esso praticata sarebbe una grave limitazione della sovranità del popolo negato nella sua qualità di pouvoir constituant. «Concettualizzato come una specie di organo costituzionale, il popolo ha soltanto "competenze determinate, assegnategli dalla Costituzione", il cui superamento significherebbe

11 Maus $(1994,79)$. La Maus tiene a precisare che l'affermazione di valori metapositivi o pre-statali non avvicina la sua posizione al «comunitarismo», il cui riferimento a strutture oggettive di socializzazione confonde aspetti normativi e descrittivi. «E vero che l'agire comunicativo e le forme della fondazione delle norme si esercitano solo in una comunità concreta, ma i valori reali di una comunità reale non possono essere criterio bensì soltanto oggetto di verifica discorsiva».

12 Preuss (1994a) presenta una diversa tipologia del rapporto tra Costituzione e sistema politico rispettivamente inglese, americano e francese, da cui discendono ovviamente differenti prospettive. Il «costituzionalismo» britannico sarebbe genuinamente politico, quello americano soprattutto sociale, mentre quello francese sarebbe sostanzialmente statalista. 
l'abolizione dello Stato costituzionale. Lungi dall'avere una "competenza delle competenze" pre-statuale per una qualsiasi decisione, il popolo è addomesticato a "grandezza costituzionalizzata"» (ibidem, 50). La Costituzione diventa fondamento e nucleo di un ordine legale in cui i diritti d'azione, adesione e di resistenza dell'individuo non sono valori pre-statuali ma «istituti giuridici»: non precedono un ordine legale fondandolo, ma ne derivano.

La conseguenza di tutto ciò è la Justitialisierung der Politik. La politica (la decisione politica che va oltre la routine amministrativa) viene praticata attraverso la interpretazione sempre più estensiva della legge costituzionale. Alla fine però il voler trovare una risposta costituzionale ad ogni questione politica produce un clima di generale insicurezza del diritto.

Come si vede, nella analisi della Abromeit ci sono punti di convergenza con Isensee - nella critica all'attivismo della Corte costituzionale e nella presa di distanza dalla concezione «sostanzialista» del dettato costituzionale. Ma quest'ultimo punto è oscuro nelle sue conseguenze, come rimane incerto anche l'esito pratico della riaffermazione di principio del pouvoir constituant del popolo. Faccio un esempio: un modello di «sovranità popolare» di tipo svizzero risolverebbe nel senso desiderato dalla autrice il conflitto d'opinione che si è creato nell'estate del 1995 attorno alla «sentenza del crocefisso»? ${ }^{13}$ Oppure proprio questo episodio che ha visto la mobilitazione del «popolo bavarese» contro Karlsruhe non sta a dimostrare quanto sia problematico il ricorso al popolo - non solo in piazza ma persino nella sua rappresentanza parlamentare quando questa può legiferare senza un superiore controllo costituzionale? Perché la sinistra radicaldemocratica ha stigmatizzato la mobilitazione popolare bavarese come un fenomeno «populista» anziché vedervi un appello al pouvoir constituant? Evidentemente la distinzione/articolazione tra potere costituente e potere costituito è nella realtà politicocostituzionale assai più complicata di quanto non appaia.

13 Nell'agosto 1995 la Corte costituzionale federale dava ragione ad un genitore bavarese che esigeva la rimozione del crocefisso dall'aula di una scuola pubblica, dopo che il tribunale costituzionale bavarese aveva respinto la sua richiesta. La sentenza della Corte di Karlsruhe ha provocato in Baviera una straordinaria ondata di proteste perché percepita come una prevaricazione contro il «popolo» bavarese e il suo diritto (costituzionalmente riconosciuto) di dare libera espressione pubblica alla sua identità religiosa. 


\section{Il custode della Costituzione di una società civile}

Proprio commentando la «sentenza del crocefisso» Günter Frankenberg parla della Corte come del Custode della Costituzione di una società civile, facendo alcuni interessanti rilievi sulla natura della sua autorità (1996). Per poter svolgere il suo ruolo, che presuppone l'esecuzione delle proprie sentenze senza disporre di strumenti esecutivi e/o coercitivi, la Corte gode di un tipo di autorità che rientra nel terzo tipo di potere legittimo messo a punto da Max Weber, quello legale-razionale. Ė la più impegnativa delle forme di autorità. Nel nostro caso la «legalità degli ordinamenti stabiliti e del diritto di dare direttive» ${ }^{14}$ su cui tale autorità si basa è quella della Costituzione scritta e i chiamati (Berufene) ad esercitarla sono i giudici della Corte. «All'inizio c'è il testo: la Legge fondamentale. Esso assegna alla Corte "il potere giurisdizionale". Tocca ai giudici, educati e informati dalla Legge fondamentale, scoprire i legami tra la legge costituzionale, i diritti fondamentali e il complesso di leggi che sono necessarie ad una società».

Alla base di questo atteggiamento - come sappiamo - c'è l'assunto che la Costituzione sia un «ordinamento di valore oggettivo» che soltanto i giudici sono autorizzati ad interpretare. In questo modo però la Corte assomiglia sempre più «pericolosamente ad una istanza ultima che si erge sopra la stessa legge costituzionale». L'unico correttivo a questa situazione è la trasparenza nella scelta dei giudici e la chiarezza delle motivazioni dei loro pronunciamenti. «Istituzionalmente indipendente e nello stesso tempo esposto all'intervento del legislatore riguardo alle sue competenze e procedure, tribunale sopra e sotto alla legge costituzionale, non organo supremo della Costituzione eppure suo controllore, amministratore del diritto ma insieme decisore politico - il Bundesverfassungsgericht in forza di questi paradossi e tensioni dovrebbe risultare minato nella sua autorità, diventando fonte di costante insicurezza istituzionale. A quanto pare invece è vero il contrario. Poco toccati dalla disaffezione (forse soltanto asserita) dei cittadini verso la politica e verso la giustizia e collocati sopra i litigi dei partiti, i 16 giudici

14 «Glaube an die Legalität gesatzter Ordnungen und des Anweisungsrechts der durch sie zur Ausübung von Herrschaft Berufenen». Nella traduzione italiana: «credenza nella legalità di ordinamenti stabiliti e del diritto di comando di coloro che sono chiamati ad esercitare il potere» (Weber 1961, 210). 
costituzionali ${ }^{15}$ godono nelle loro sentenze di una considerazione (s)proporzionatamente alta - accanto al cancellierato e al Parlamento - a dispetto delle proteste ricorrenti».

Il Bundesverfassungsgericht non va dunque né idealizzato né demonizzato: la sua autorità si fonda in definitiva sulla capacità di gestire il conflitto. Questo significa che deve istaurare una prassi comunicativa sempre aperta, controversa e controvertibile, in un processo autoriflessivo nel quale la Costituzione equivale ad una «convenzione fondativa» (grundlegende Konvention). Così Frankenberg chiama «l'insieme di regole conflittuali dovute ad un accordo esplicito o tacito, sempre modificabile, segnalabile nella prassi sociale».

La formula «convenzione fondativa» è suggestiva ma - a mio avviso - è troppo indeterminata rispetto alla sostanza delle questioni sollevate. In definitiva si limita a invocare una sorta di primato della politica intesa come ricerca sempre aperta e negoziata di regole.

Al primato della politica approdano anche le riflessioni di Ernst-Wolfgang Böckenförde su democrazia e Costituzione. Tale primato coesiste tuttavia con la regola della astinenza o della «riservatezza in politicis» da parte dei componenti della Corte. Come criterio di comportamento per il giudice costituzionale c'è solo l'etica professionale. «Nessuno, salvo il giudice stesso con il suo ethos e senso di responsabilità, può offrire la garanzia di esercitare il suo ufficio come lo richiedono la Costituzione e la legge. Qui si rivela un nesso sistematico tra diritto e morale» ${ }^{16}$. E - aggiungiamo noi - il paradosso tra politicità della giustizia costituzionale e apoliticità dei giudici.

Fatta questa premessa, ci si chiede come dall'affermazione della Costituzione intesa quale «ordinamento di valori» oggettivo possa discendere un ruolo attivo della Corte nell'interpretazione di tale ordinamento. Vediamo in proposito alcune considerazioni di Böckenförde tolte dalla sua raccolta di scritti di teoria e diritto costituzionale Staat, Verfassung, Demokratie.

In Germania l'idea della Costituzione come ordine della vita in forma giuridica (rechtliche Lebensordnung) e fondamento di valore (Wertgrundlage) della collettività politica si afferma sol-

15 Frankenberg usa l'espressione «leoni sotto il trono» secondo una immagine spesso usata per $i$ giudici costituzionali americani. La versione italiana dell'intera citazione è un po' semplificata rispetto all'originale.

16 Così nel citato articolo comparso sulla «Frankfurter Allgemeine Zeitung». 
tanto dopo la prima guerra mondiale, di fronte ai potenziali di disintegrazione sociale e morale della Repubblica di Weimar. Attraverso il diritto si cerca di assicurare quei valori di fondo del vivere collettivo che ne fanno una autentica «comunità» politica. «In questo modo alla Costituzione viene assegnato un significato costitutivo non solo per l'ordinamento del potere politico, ma per l'ordine giuridico della vita della comunità nel suo insieme» (Böckenförde 1991, 48). Dopo la catastrofe del Terzo Reich, questa idea viene ripresa e ribadita dagli estensori del Grundgesetz del 1949. Da parte sua la Corte federale dichiara parecchie volte esplicitamente che la Costituzione attraverso i diritti fondamentali contiene un «ordine valoriale» oggettivo, un «sistema di valori» identificabile e una «decisione di valore».

Questa concezione valoriale oggettiva della Costituzione investe tutti gli àmbiti della vita sociale con un sovraccarico di attese e pretese di giurisdizione non privo di inconvenienti. I valori infatti - osserva Böckenförde - sono tali soltanto in quanto «valgono di fatto» e sono destinati ad autoaffermarsi continuamente per non sparire. $\mathrm{Ma}$

una conoscenza razionalmente controllabile dei valori e di un ordine di valore non esiste, come non esiste un sistema di valutazione e di preferenza di valori razionalmente controllabile per le pretese di validità concorrenti di valori diversi, spesso in collisione tra loro (...). Il richiamo ai «valori» non è nulla di più che una formula pluralistica unificante per qualcosa che ha bisogno di una fondazione di senso in vista della fondazione dell'ordine statale e sociale, senza che questa fondazione sia già data con questo richiamo. I «valori» non sono altro che un modo per designare il consenso esistente o postulato. Dal punto di vista giuridico la concezione della Costituzione come fondamento di valore e ordine di valori non fornisce la fondazione metapositiva desiderata, ma apre piuttosto all'afflusso di visioni e postulati di consenso mutevoli la strada all'interpretazione e applicazione della Costituzione. Con ciò il suo contenuto normativo è sottoposto alle correnti del tempo di volta in volta dominanti (ibidem, 51-52) ${ }^{17}$.

Questo passaggio merita di essere riportato per esteso nella sua complessità perché lascia trasparire un «politeismo dei valori» della Costituzione che non è lontano dal relativismo. Non c'è dubbio che l'autore non gradirebbe questa osservazione:

17 Un tesi analoga si ritrova nella conclusione di Böckenförde (1996b, 252): «Il richiamo ai valori o valori fondamentali, oggi diffuso, non contribuisce per niente alla stabilizzazione. I "valori" infatti sono una categoria del valere (Gelten); essi definiscono in modo molto astratto un consenso presente o postulato ma non sono in grado di fondarlo da sé». 
protesterebbe che lui non mette affatto in dubbio la certezza dei diritti fondamentali garantiti dalla Costituzione; semplicemente dubita che su di essi si possa costruire una dogmatica giuridica definita e valida una volta per tutte ${ }^{18}$.

$\mathrm{Ma}$ facciamo un passo in avanti. Se i diritti fondamentali hanno il carattere di norme di principio, richiedono qualcosa di più di una esegesi del testo costituzionale: impongono un intervento creativo di legge che diventa vincolante per lo Stato. Muta così il rapporto tra legislazione normale e giurisdizione costituzionale. Entrambe creano diritto ma in maniera concorrenziale: la legislazione normale ha la precedenza, ma la giurisdizione costituzionale possiede un rango superiore.

In questo modo la giustizia costituzionale promuove il passaggio dallo Stato legislativo parlamentare allo Stato giurisdizionale. Questa trasformazione ha luogo appunto grazie alla interpretazione dei diritti fondamentali come norme di principio oggettivo e al riconoscimento della esclusiva competenza della Corte costituzionale per la loro concretizzazione.

Il compito della Corte cambia da applicazione giurisprudenziale del diritto a giurisdizione costituzionale, nel senso antico che precede la separazione tra produzione del diritto e applicazione del diritto (...). La Corte diventa un forte organo politico (non partitico), un areopago della Costituzione; quel lembo di sovranità che tiene tra le mani in forza della sua competenza per l'ultima decisione vincolante, si amplia. Nasce così l'interrogativo circa la legittimazione democratica della Corte (Böckenförde 1991, 190-191).

Appare così il nesso stretto esistente tra l'alternativa Stato parlamentare-Stato giurisdizionale e la questione del vincolo giuridico oggettivo dei diritti fondamentali.

Chi vuole tener ferma la funzione-guida del Parlamento nella produzione del diritto ed evitare ogni ulteriore passo nella costruzione di uno Stato giurisdizionale dei giudici, deve anche tenere ferma la tesi che i diritti fondamenta-

18 Confesso che non mi è chiara in Böckenförde la rilevanza del nesso tra la sua posizione «relativista» sui valori e il restante discorso giuridico. Un costituzionalista italiano, Gustavo Zagrebelsky, sviluppa un ragionamento antitetico quando parla di ancorare l'attività del legislatore «a un insieme di valori e principi costituzionali superiori sui quali, malgrado tutto, si realizza un sufficientemente ampio consenso sociale. Il pluralismo non degenera in anarchia normativa a condizione che, malgrado la divisione sulle strategie particolari dei gruppi sociali, vi sia una convergenza generale su alcuni aspetti strutturali della convivenza politica e sociale e si possano così mettere fuori discussione e consacrarli in un testo non disponibile da parte degli occasionali signori della legge e delle fonti concorrenti con la legge» $(1992,48)$. Su questa intera problematica si veda Dogliani (1996). 
li - legalmente esigibili - sono «soltanto» diritti soggettivi di libertà di fronte al potere statale e non anche norme oggettive (vincolanti) per tutti gli ambiti del diritto (ibidem, 194).

Si configura così un'altra alternativa: quella tra una Costituzione come ordine-quadro (Rabmenordnung) o una Costituzione direttiva (dirigierende Verfassung). Nel primo caso essa non contiene già tutto il materiale che concretizza e armonizza le diverse posizioni del diritto individuali, ma pone solo dei «paletti» di carattere difensivo. Nel secondo caso la Costituzione fonda l'ordine giuridico complessivo della società. Nel primo caso la Corte non può «riempire» il quadro costituzionale con tutte le pretese degli individui. Nel secondo caso, invece, la Corte con il suo lavoro di attiva concretizzazione dei diritti individuali diventa «padrona della Costituzione».

In questo contesto Böckenförde sembra più preoccupato di mettere a fuoco un problema che non di sostenere la tesi che nello Stato costituzionale democratico i diritti umani fondamentali non possono considerarsi meri diritti soggettivi bensì norme di valore oggettivo che diventano «compiti dello Stato» democratico. Si limita a riaffermare il primato della politica, portando l'attenzione sul cittadino e sulle sue possibilità di scelta: il cittadino si affiderà al legislatore eletto in Parlamento o alla Corte costituzionale? Di fronte a questo interrogativo di carattere squisitamente politico, al giudice personalmente non rimane che l'apoliticità dell'etica professionale.

Per quanto ineccepibile, questa soluzione mi pare inadeguata rispetto alla vastità dei problemi sollevati. Il paradosso (di cui parlavamo sopra) in cui si trova la Corte nel prendere le sue decisioni, non trova una soluzione soddisfacente. Non c'è dubbio che la Corte nell'interpretare il proprio compito di «custode della Costituzione» segue una logica autoreferenziale - come osserva sempre Böckenförde. Ma occorre aggiungere che i giudici costituzionali non rimangono estranei ai dibattiti che investono il mondo della giustizia - dibattiti innescati da questioni sociali, etiche e politiche che influiscono direttamente o indirettamente sulla formazione dei criteri di giudizio dei membri stessi della Corte. L'immagine della Corte costituzionale come turris eburnea, presidiata dall'etica professionale, può lusingare qualche suo inquilino, ma è una pura finzione. Rimane aperto il quesito se «il discorso giuridico-costituzionale» sia in grado di surrogare o addirittura di interpretare il «discorso 
politico» che si istaura (o dovrebbe istaurarsi) nella società civile tra i cittadini ${ }^{19}$.

Quali conseguenze hanno queste considerazioni nel problema europeo?

\section{La Corte tedesca pone dei limiti di competenza all'Unione europea}

È noto che il trattato di Maastricht, oltre ai famosi criteri di convergenza economico-finanziaria in vista della moneta unica che hanno monopolizzato l'attenzione dei governi e dei commentatori, ha ribadito la necessità che la politica comunitaria sia «vicina ai cittadini» con l'ampliamento delle prerogative del Parlamento europeo e il rafforzamento dell'influenza dei livelli regionali e locali; con la riaffermazione del principio della sussidiarietà e una maggiore attenzione alla identità nazionale dei singoli Stati membri. Il tutto sotto il segno del primato dei diritti umani fondamentali.

$\mathrm{Ma}$ non è chiaro se e come questo complesso di raccomandazioni incida sulla struttura politica dell'Unione europea, rispetto alla quale il trattato si è espresso in modo molto cauto parlando di «unione sempre più stretta dei popoli d'Europa». È opinione condivisa dalla quasi totalità degli scienziati politici e del diritto che l'Europa non può concepirsi come una nazione né tanto meno come uno Stato. La cittadinanza europea che è scritta sui passaporti degli abitanti del vecchio continente (anzi soltanto di alcuni di essi), non certifica e non crea alcun «popolo europeo» inteso nel senso forte di soggetto fondativo e attivo di politica (uno Staatsvolk), quale è celebrato nelle Costituzioni nazionali. Che cosa è allora l'Unione europea?

Il Bundesverfassungsgericht nella sua sentenza del 12 ottobre 1993 sul trattato di Maastricht si limita a dire che «il trattato dell'Unione fonda una associazione di Stati (Staatenverbund) per la realizzazione di una unione sempre più stretta dei popoli

19 A questo proposito Portinaro ricorda che «l'idea habermasiana della Corte come "custode della democrazia deliberativa" e quella rawlsiana della stessa come "paradigma della ragione pubblica" si alimentano della stessa diffidenza nei confronti di possibili involuzioni paternalistiche della giustizia costituzionale, nella convinzione che $i$ discorsi giuridici possano sviluppare strategie interpretative capaci di favorire nel circuito decisionale il momento discorsivo ma "non possano sostituirsi ai discorsi politici che servono alla fondazione delle norme e dei programmi e devono sempre presupporre l'inclusione di tutti gli interessati" (Habermas)» (1996, 30-31). 
d'Europa - organizzati statalmente - non uno Stato poggiante su un popolo (Staatsvolk) europeo». Colpisce il fatto che la Corte abbia sentito la necessità di quest'ultima precisazione negativa. In altra parte della sentenza l'Unione viene presentata come una «istituzione interstatale» (zwischenstaatliche Einrichtung) sia pure con caratteristiche e vincoli molto peculiari.

Attorno a queste definizioni si è aperta una discussione molto impegnativa ${ }^{20}$. La sentenza di Karlsruhe ha il grande merito di porre la questione della statualità a dimensione europea, costringendo scienziati politici e costituzionalisti a misurarsi con questo problema. Ricordo, a questo proposito, che in vista della ratifica del trattato, il Grundgesetz tedesco ha subito importanti innovazioni e mutamenti. Tra le più consistenti va segnalata la sostituzione del testo del vecchio art. 23 con uno completamente nuovo che menziona espressamente l'obiettivo di una Europa unita e il relativo trasferimento di diritti di sovranità. Ribadisce nel contempo la necessità che ci sia piena conformità dei principi ispiratori dell'Unione (diritti fondamentali, democrazia, Stato di diritto, federalismo, ecc.) con quelli già garantiti dalla Legge fondamentale della Bundesrepublik ${ }^{21}$. Sulla base di questa esigenza la Corte tedesca ha ispirato la sua sentenza su Maastricht alla preoccupazione che la istituzionalizzazione dell'Unione europea risponda ad autentici criteri democratici. Contestualmente emergono i quesiti sulla possibile statualità della sua forma politica e sulla qualità del trasferimento di sovranità da parte degli Stati membri.

$\mathrm{Al}$ centro del problema c'è dunque la democrazia o più precisamente la sua legittimazione istituzionale. Questa, secondo la sentenza della Corte, può essere garantita soltanto dall'abbinamento (Rückkoppelung) dell'azione degli organi europei a quella dei parlamenti nazionali. «Se - come accade oggi - sono i popoli a fornire la legittimazione democratica attraverso i parlamenti nazionali, il principio democratico pone dei limiti all'estensione dei compiti e delle prerogative delle comunità euro-

20 Nella impossibilità di segnalare la vastità del dibattito mi limito a segnalare due saggi ricchi di riferimenti alla letteratura: Frowein (1994) e Weiler (1995).

21 La nuova versione dell'art. 23 recita tra l'altro: «Per la realizzazione di un'Europa unita la Repubblica federale di Germania collabora allo sviluppo dell'Unione europea che deve osservare il principio democratico, dello Stato di diritto, sociale e federativo e il principio di sussidiarietà e deve assicurare una tutela dei diritti fondamentali essenzialmente paragonabile a quella della presente Legge fondamentale. Il Bund può a questo scopo, mediante legge, con l'assenso del Bundesrat, trasferire diritti di sovranità». 
pee. Al Parlamento tedesco devono rimanere compiti e prerogative di peso sostanziale».

Questa dichiarazione si presta a letture differenti. Da un lato, infatti, la sentenza prende atto che a tutt'oggi le istituzioni europee sono largamente deficitarie in fatto di democrazia. $\mathrm{Ma}$ lungi dal premurarsi che questo deficit possa essere superato con l'ampliamento delle competenze del Parlamento europeo, la Corte ribadisce che, stando così le cose, i parlamenti nazionali devono mantenere competenze sostanziali di non piccolo peso. Con ciò viene riaffermato il principio della statualità (degli Stati membri) come unica fonte della legittimità democratica dell'Unione con il sottinteso della necessaria omogeneità (relativa) dei popoli (Staatsvölker) ${ }^{22}$.

Detto questo, la Corte non abbraccia le tesi di chi, usando argomenti analoghi, esclude senz'altro in linea di principio che l'Unione possa subire un'evoluzione rispetto allo status quo. Particolarmente significativo è il fatto che la Corte non ritenga che l'assenza di una lingua comune, di un'opinione pubblica comune e di un sistena politico comune in Europa sia un motivo sufficiente per negare a priori la legittimazione democratica dell'Unione o un processo che in prospettiva superi questi bandicaps. Anche se esclude l'esistenza di uno Staatsvolk europeo, vede nella cittadinanza dell'Unione un «vincolo giuridico durevole» che «pur non possedendo una consistenza paragonabile alla appartenenza ad uno Stato nazionale, dà espressione giuridica sufficiente a quanto già esiste di esistenzialmente comune». Per il rafforzamento di questo vincolo valgono le raccomandazioni per un sistema elettorale europeo omogeneo, per un sistema partitico convergente e per tutte quelle misure che facciano sì che il cittadino elettore possa comunicare nella sua lingua con l'autorità cui è sottoposto.

Sin tanto che sono gli Stati nazionali e i loro parlamenti a fornire la legittimità democratica all'Unione europa - prosegue la sentenza della Corte - un «sovraccarico» di compiti e competenze di quest'ultima «indebolirebbe permanentemente la de-

22 Nella sentenza si precisa: «Ciascuno dei popoli è il punto di partenza di un potere statale ad esso riferito. Gli Stati hanno bisogno di propri campi di azione sufficientemente importanti nei quali il singolo popolo si possa sviluppare e articolare in un processo di formazione della volontà politica, da esso legittimato e guidato, per dare espressione giuridica a ciò che lo lega spiritualmente, socialmente e politicamente - in modo relativamente omogeneo». 
mocrazia a livello statale». Anche se il trattato di Maastricht non toglie al Bundestag competenze di decisione e di controllo al punto da pregiudicare il principio democratico stabilito dal testo costituzionale, ci sono dei limiti all'ampliamento ulteriore delle competenze dell'Unione. Da più parti ci si è chiesto in che cosa debbano consistere esattamente questi limiti.

In una sola frase - ha osservato Ingolf Pernice - la Corte lega l'idea dei limiti con la trasmissione della legittimazione democratica attraverso gli Stati nazionali. Ne segue che tali limiti non hanno motivo d'essere quando la sovranità fosse direttamente legittimata dai popoli europei? La giustificazione consiste, per il resto, in un postulato di teoria dello Stato che non lascia vedere né un collegamento di carattere costituzionale, né le dimensioni consentite dell'allargamento dei compiti. In fondo rimane solo la banale affermazione che gli Stati membri come Stati possono fornire legittimazione democratica sin che non hanno perso la loro qualità di Stato $(1995,267)$.

In realtà, quando parla di limitazione dei compiti dell'Unione la Corte si oppone anticipatamente ad una «estensione» dei patti stipulati tra gli Stati membri che sia di competenza della sola Unione. Su questo punto la sentenza è esplicita nel dichiarare come «non vincolanti nell'ambito della sovranità tedesca» atti giuridici che dovessero superare tali limiti. Segue una frase inequivoca: «Di conseguenza, la Corte costituzionåle verifica se atti giuridici delle istituzioni e degli organi europei rimangono nei diritti di sovranità loro assegnati o li travalicano». Questa riserva di controllo dei diritti di sovranità (Hobeitsrechte) fa pensare ad una anticipata ricusazione dell'autorità della Corte europea di giustizia, che è stata istituita apposta per verificare se gli atti giuridici degli organi dell'Unione vanno oltre le loro competenze. Se è così, ci troveremmo di fronte ad una presa di posizione grave. Non resta che attendere l'effettivo configurarsi di una controversia tra la Corte di Lussemburgo e quella di Karlsruhe.

Viene il sospetto tuttavia che la preoccupazione della Corte per i diritti di sovranità tedesca abbia un obiettivo più circoscritto anche se cruciale: la stabilità della moneta come «dovere costituzionale specifico della Repubblica federale» ${ }^{23}$. Non si

$23 \mathrm{La}$ Corte tiene a precisare che «la Repubblica federale con la ratifica del trattato dell'Unione non si sottomette ad un "automatismo" verso l'unione monetaria, impenetrabile, non più governabile nel suo svolgimento; il trattato apre la strada ad una ulteriore graduale integrazione della comunità giuridica europea che in ogni suo successivo passo dipende dai presupposti oggi prevedibili per il Parlamento o dal consenso del governo federale ulteriormente influenzabile dal Parlamento». 
tratta di una presa di posizione estemporanea ma della ripresa di un articolo della Costituzione (l'art. 88), introdotto in previsione della creazione della Banca centrale europea cui sono demandate alcune prerogative della Bundesbank.

$\grave{E}$ inutile ricordare in questa sede quanta ansia e contrasto politico sollevi in Germania il progetto di abbandonare il marco per la nuova moneta europea. È comprensibile che la Corte si faccia portavoce di queste ansie ricordando il dettato costituzionale per quanto esso sia generico ai fini di una effettiva politica monetaria. Ma a ben vedere, se è problematica l'indicazione generale dell'imperativo della «stabilità dei prezzi», non lo è affatto il suo presupposto politico: soltanto la Germania può decidere sulla propria moneta. In questa ottica, diventa irrilevante il fatto che la stabilità della moneta in Germania sia garantita da una istituzione, la Bundesbank, il cui indirizzo operativo non dipende da meccanismi di legittimazione democratico-parlamentari. L'obiettivo della stabilità monetaria è così importante per il Bundesverfassunsgericht che da «dovere costituzionale» (ai sensi dell'art. 88 del Grundgesetz) diventa «dovere di lealtà» reciproca tra i membri della Comunità (ai sensi dell'art. 5 del trattato comunitario), al punto da non escludere l'ipotesi di uno scioglimento del trattato stesso.

\section{Una Costituzione per l'Europa? Argomenti pro e contro}

«Il principio democratico non impedisce alla Repubblica federale di Germania di essere membro di una comunità interstatale - organizzata in modo sopranazionale. Il presupposto di tale partecipazione tuttavia è che sia assicurata una legittimazione proveniente dal popolo e la sua influenza anche all'interno dell'associazione degli Stati». Così leggiamo ancora nella sentenza della Corte. La definizione dell'Unione europea come di una «comunità interstatale, organizzata in modo sopranazionale» esclude che essa possa dotarsi di una Costituzione? $\mathrm{E}$ difficile dirlo: la risposta dipende anche da che cosa la Corte intende per Costituzione.

Il testo più noto che affronta in modo critico la questione di una possibile Costituzione europea è quello di Dieter Grimm, Braucht Europa eine Verfassung?24. Si tratta di uno scritto che

24 «L'Europa ha bisogno di una Costituzione?». Si tratta della relazione tenuta da 
sintetizza quasi tutti gli argomenti giuridici che gettano dubbi sulla consistenza e sulla augurabilità di una Costituzione europea. I punti forti dell'argomentazione sono sostanzialmente due: la statualità (Staatlichkeit) quale requisito per ogni costruzione costituzionale e il carattere già «costitutivo» dei trattati interstatali che sono all'origine della Comunità/Unione europea e delle sue competenze. Ebbene, la qualità di Stato manca all'Unione europea, che si presenta come semplice comunità giuridica (Rechtsgemeinschaft) mentre, d'altro lato, sono i trattati interstatali a costituire la fonte esclusiva del diritto comunitario primario. Rimane aperta la questione se il diritto comunitario primario stesso possa definirsi già come una Costituzione come ritiene la scienza del diritto europeo - oppure valga la tesi che la forma del vincolo giuridico del potere qualificante la Costituzione debba essere riconosciuta sempre soltanto agli Stati contraenti $\mathrm{i}^{25}$. Naturalmente i trattati non sono l'equivalente della Costituzione tout court, perché c'è un'enorme differenza nel tipo di volontà politica da cui emanano. I trattati rimandano ai governi degli Stati membri, la Costituzione emana dal popolo e quindi nel caso europeo dovrebbe emanare dal «popolo dell'Unione». A tutt'oggi «il potere pubblico europeo non deriva dal popolo ma è trasmesso mediante gli Stati (...). Nella misura in cui nella Costituzione è in gioco la legittimazione del potere da parte di chi vi è sottoposto, i trattati le rimangono indietro» ${ }^{26}$. Si apre così quello che Grimm chiama uno «iato democratico»: il principio democratico viene fatto valere nell'Unione europea per il tramite degli Stati membri proprio nel momento in cui questi vedono diminuire le proprie competenze decisionali. Viceversa i poteri decisionali dell'Unione crescono mentre rimane debole il suo principio democratico. Da qui il bisogno di una legittimazione della politica europea non derivata dai governi degli Stati-membri.

Ma questo bisogno di legittimazione riporta alla problemati-

Dieter Grimm alla fondazione Carl Friedrich von Siemens il 19 gennaio 1994 e pubblicata a cura della stessa Fondazione. Una versione italiana compare in Zagrebelsky, Portinaro e Luther (1996). Qui ho preferito seguire la versione originale.

25 «Rispetto al potere pubblico dell'Unione europea i trattati assumono le funzioni essenziali che all'interno dello Stato competono alla Costituzione. Nella misura in cui la Costituzione è la giuridificazione del potere politico, i trattati non lasciano nulla a desiderare. Le esigenze fondamentali del costituzionalismo moderno sono assolte nella Comunità» (Grimm 1994, 32).

26 Ibidem (47). 
ca delle (pre)condizioni della democrazia. Al primo posto c'è la questione della lingua, dell'opinione pubblica, della comunicazione e del discorso politico a livello europeo. Sin tanto che questo nodo rimane irrisolto, la piena parlamentarizzazione dell'Unione europea, sul modello dello Stato costituzionale nazionale, rimane un traguardo insufficiente. Ciò che ostacola la democrazia europea è

la debole identità collettiva e la scarsa capacità discorsiva sopranazionale. Questo significa che il deficit di democrazia europea è strutturale. E non può essere superato a breve termine neppure con le riforme istituzionali. In queste circostanze la trasformazione dell'Unione europea in uno Stato federale non è un obiettivo auspicabile a breve termine ${ }^{27}$.

Affermare questo non significa privilegiare lo Stato nazionale, che non è più all'altezza dei suoi compiti, ma riconoscere che l'indispensabile convergenza e cooperazione tra gli Stati non porta necessariamente ad una unità statale. L'Unione europea deve mantenere «la sua specificità di istituzione sopranazionale senza copiare modelli statali nazionali».

E su questo sfondo di problemi che si colloca il tema della Costituzione europea. Le riforme istituzionali necessarie per far fronte all'allargamento dell'Unione non richiedono di per sé una Costituzione sul modello degli Stati nazionali. Se attraverso un formato costituzionale si vogliono mettere in evidenza, a beneficio dei cittadini, i fondamenti comuni di diritto dell'Unione, basta tener distinte le varie e numerose regolamentazioni comunitarie da un nucleo di elementi fondanti. Questo nucleo centrale del contratto comunitario (Kernvertrag) può essere assimilato nel suo modo di presentarsi (Erscheinungsbild) ad una Costituzione, senza togliere nulla al suo carattere pattizio.

Se invece si volessero aggiungere ai trattati quegli elementi che loro mancano per diventare una Costituzione nel senso pieno del concetto, allora si andrebbe inevitabilmente verso la statalizzazione dell'Unione. Questo esito statalista deve essere ben chiaro a coloro che chiedono a gran voce la Costituzione europea per mera simpatia verso questa idea o in nome della democrazia. Quello che manca ai trattati perché siano una vera e propria Costituzione è

la legittimazione popolare dell'atto giuridico costitutivo dell'Unione e l'auto-

27 Ibidem (49-50). 
determinazione dei cittadini dell'Unione circa la forma e il contenuto della sua unità politica. Ma se si facesse questa operazione si muterebbe il fondamento di legittimazione dell'Unione europea (...) Il costituente sarebbe il popolo dell'Unione ${ }^{28}$.

Solo con questo atto l'Unione potrebbe pretendere la competenza delle competenze (die Kompetenz-Kompetenz) e la somma dei suoi poteri non sarebbe più condizionata dal trasferimento degli Stati membri. Ma tutto questo - ripete Grimm significa la creazione di uno Stato. Uno Stato per altro che, non disponendo delle condizioni di intermediazione democratica, sarebbe più che mai lontano dai suoi principi. In queste condizioni una Costituzione europea non potrebbe superare «lo iato democratico», di cui si parlava sopra, e andrebbe incontro ad una grave disinganno.

Una posizione scettica nei confronti della Costituzione europea è sostenuta anche da Josef Isensee, con argomenti simi$\mathrm{li}^{29}$. Creata dal diritto che deriva dai contratti e tenuta in vita dai contratti, l'Unione europea non ha bisogno di nessuna Costituzione improntata sul modello statale - dal momento che ha già un suo statuto legale. Questa tesi ha il suo punto di forza nell'assunto che presupposto della Costituzione è lo Stato. E l'identità dello Stato come soggetto di diritto rimane anche quando la Costituzione muta.

E vero che il Grundgesetz nel suo preambolo del 1949 e ancora nella modifica dell'art. 23 del 1992 parla di «realizzazione di una Europa unita», e l'art. 24 autorizza il legislatore a trasferire alcuni diritti di sovranità. $\mathrm{Ma}$ non si tratta - scrive Isensee - del trasferimento del potere statale in blocco. Oltretutto esso avviene a beneficio di «istituzioni interstatali» non di uno Stato sopranazionale. Insomma, il dettato costituzionale che obbliga la Germania alla realizzazione dell'Europa unita non ha di mira la creazione di uno Stato europeo.

Vorrei fare un solo appunto al modo di argomentare di Isensee, che è esemplare per molti altri euroscettici. Di fronte

28 «Questo vale anche se alla Costituzione e al suo cambiamento partecipassero gli Stati membri. Lo farebbero non più come "padroni dei trattati" ma - in analogia al Bundesrat tedesco - come organo dell'Unione che si autodetermina».

29 «Che non esista un popolo dell'Unione (Unionsvolk) che possa fornire la base di legittimazione alla democrazia europea, non significa di per sé un allontanamento dal concetto di Stato di diritto internazionale, ma un allontanamento dal concetto costituzionale democratico. Il concetto di Stato è indifferente alla Costituzione, anche alla democrazia. Si fonda non sulla legittimità ma sulla effettività» (Isensee 1995, 573). 
all'idea della «statualità aperta» e dello «Stato costituzionale cooperativo» che è implicita nelle dichiarazioni di intenti del Grundgesetz in prospettiva europea, Isensee diventa contorto: parla della sovranità dello Stato nazionale che «si afferma» nel momento stesso in cui «limita se stessa» o della statualità che «si relativizza» proprio quando «si conferma e si allarga» nella sua efficacia attraverso i vincoli sopranazionali, ecc. Questo stile ellittico riflette involontariamente quella originalità del costrutto europeo di cui gli euroscettici sanno cogliere soltanto gli aspetti negativi.

Chi si pone in una prospettiva positiva verso la Costituzione europea deve saper argomentare in modo diverso, puntando ad esempio sulla «società civile europea» o sui nuovi compiti che gli Stati democratici europei devono assumersi per l'attuazione dei diritti umani fondamentali. Questo è il «vero tema europeo» - dice Erhard Denninger in occasione di un simposio berlinese dedicato a questi problemi $(1996)^{30}$. E critica la posizione di chi sostiene l'impossibilità di una Costituzione europea, esclusivamente in base alla tesi della natura contrattuale (interstatuale) dell'Unione europea e della priorità della forma-Stato. Questo ristretto modo di ragionare rimane legato all'idea di Stato-nazione e di popolo ottocenteschi ed elude l'interrogativo «se e come possa nascere una società civile europea». Con particolare fermezza l'autore contesta l'esistenza di una statualità di base che precede la Costituzione giuridica. «L'idea di una statalità come strumento di potere, razionale allo scopo, ma indifferente al fine, con compiti neutrali non è né premoderna, né moderna - è un'astrazione irreale astorica». Storicamente il potere statale è sempre stato legato in qualche modo ad un potere della legge. Denninger, pur riconoscendo le difficoltà del formarsi e del crescere di un «repubblicanesimo europeo», è convinto che ci siano le condizioni per una «sfera pubblica in grado di condurre "discorsi"» (diskursfäbigen Öffentlichkeit), capace

30 «Non solo per la situazione del diritto costituzionale tedesco si constata la tendenza a considerare $\mathrm{i}$ "diritti umani" non come diritti soggettivi individuali, come diritti di ogni uomo, ma come norme oggettive di diritto, come compiti o fini dello Stato. (...) $\mathrm{La}$ "Costituzione dell'Europa", quando mai dovesse trovare espressione in un testo, nel determinare il rapporto dei diritti umani ai compiti statuali deve dare pesi diversi da quelli presenti sinora nel trend comunitario, Maastricht compresa» (Denninger 1996). Un autore che fa dei diritti fondamentali il Leitmotiv della sua riflessione è Häberle (1993; 1994). 
quindi di un nuovo e diverso fondamento giuridico-costituzionale dell'Unione europea.

A proposito di Costituzione europea Christian Tomuschat osserva che il sistema dei poteri di cui gode l'Unione potrebbe già fregiarsi della qualifica di Costituzione. Purché questo termine non venga sfruttato come «corno dell'abbondanza» per ogni tipo di deduzione.

L'Unione europea non è un vincolo d'amore (Liebesbund) ma una comunità a scopo utilitario (Zweckgemeinschaft), guidata dalla sobria convinzione che molti dei compiti che potevano essere svolti nell'ambito dello Stato nazionale, oggi nel segno della globalizzazione devono essere affrontati ad un livello più alto (1996).

\section{La politica della Costituzione europea}

Tiriamo le fila. Le difficoltà di fissare concettualmente in modo convincente la costruzione politica odierna e in fieri dell'Europa non si possono banalizzare. Dietro a quello che può sembrare un esercizio di acribia attorno alla Staatsqualität o alla Verfassungsfäbigkeit dell'Europa è in gioco la determinazione politico-istituzionale di uno dei fenomeni più importanti del nostro tempo. Che non si tratti di nominalismo lo si vede - e lo si vedrà sempre meglio in futuro - dal modo con cui la Corte costituzionale tedesca sta determinando (o cercando di determinare) competenze e regole di comportamento attraverso una circospetta operazione definitoria. Qualificare l'Unione europea come uno Staatenverbund nel tentativo di creare un tertium genus tra quelli storicamente ben definiti di Staatenbund (federazione di Stati) e Bundesstaat (Stato federale) può essere interpretato alternativamente come un atto di buona volontà europeista della Corte o come un compromesso terminologico che scherma tutt'altre intenzioni. Oppure è semplicemente il rispecchiamento di un costrutto istituzionale europeo monstro simile come Samuel von Pufendorff chiamava il Sacro romano impero della nazione tedesca? ${ }^{31}$

31 Tomuschat (1996) contesta la pertinenza di questa analogia, dal momento che il Sacro romano impero della nazione tedesca disponeva di pochissime leggi fondamentali e per il resto il diritto nasceva di volta in volta da prassi statuali. La situazione legale dell'Unione europea è completamente diversa e i problemi sono di altra natura, non foss'altro perché per tutte le competenze ci sono principi stabiliti in testi formali di leg- 
Mi chiedo se, al di là dei risultati di questo sforzo definitorio, ciò che conti non sia il fatto stesso e la qualità del coinvolgimento della Corte tedesca (ed eventualmente delle altre Corti a fianco della Corte europea) nell'abbozzo di uno Stato costituzionale di tipo particolare, già intravisto nell'evoluzione di alcuni Stati nazionali. È solo attraverso i pronunciamenti delle Corti che acquistano consistenza i discorsi sui diritti di cittadinanza, sui valori primari della società civile, su una identità plurima europea ecc.

Ma questi discorsi si fermano davanti alla definizione di una Costituzione europea. Abbiamo visto come alcuni studiosi e politici sostengano che una Costituzione europea già esiste attraverso i trattati costitutivi dell'Unione stessa. Naturalmente si tratta di una Costituzione implicita (anche se esplicitabile meglio) ma non equiparabile a quella degli Stati nazionali. Qui le opinioni divergono tra chi sdrammatizza questo dato di fatto, vedendovi appunto la novità anche costituzionale dell'Unione $\mathrm{e}$ chi lo considera invece come la prova della impossibilità (o non augurabilità) di una Costituzione europea.

$\grave{E}$ interessante l'argomento che sostiene questa tesi. A prima vista può apparire un nostalgico attaccamento alla statualità tradizionale: in realtà esprime la convinzione che il principio democratico si salvaguardi soltanto nell'ambito delle forme di legittimazione popolare consolidate dello Stato democratico esistente (i parlamenti nazionali). In questo quadro acquistano valore le osservazioni sulla inesistenza del «popolo/demos» europeo. Anche nel caso si arrivasse ad una Costituzione europea la democrazia potrebbe essere garantita soltanto con la permanenza di forme di controllo e di legittimazione radicate negli Stati nazionali. Non è vero che i sostenitori di questa posizione, etichettata dagli avversari come nazionalista e étatiste, coltivano nostalgie stataliste vecchia maniera. Non a caso il loro bersaglio preferito è proprio un «Superstato» europeo, lontano dai cittadini e dal loro controllo. Su questo punto non possono non es-

ge. Da questo punto di vista il sistema funziona. «Ma si approssima un ambito di confine, dove la collisione con lo Stato nazionale diventa quasi inevitabile. In fondo è il problema schmittiano della "situazione di emergenza" che incombe. Scoppierà ad un certo punto un conflitto che non potrà più essere risolto nelle procedure previste nei trattati? Allora e soltanto allora si può sensatamente ritornare a modelli giuridici generali che però proprio in quella situazione di tensione saranno quasi interamente sovraccaricati da considerazioni politiche». 
sere d'accordo anche gli europeisti più zelanti e convinti della necessità di una Costituzione europea ad hoc, costruita su una «statualità aperta», centrata sulla «società civile» e mirata alla costruzione di un demos europeo, attorno alla valorizzazione dei diritti fondamentali e dello status della cittadinanza.

In realtà la prospettiva di questo Stato costituzionale europeo, per quanto suggestiva ${ }^{32}$, non solo non è in grado a tutt'oggi di offrire modelli politici operativi, ma si muove ancora con grande genericità e incertezza nei codici della cittadinanza, della società civile, dell'identità plurima ecc. Di questa debolezza risentono innanzitutto i discorsi sulla Costituzione che - è bene sottolinearlo - non è un semplice catalogo di norme fondamentali plasmato su grandi valori ma è un vincolo di obbligazione politica, che per farsi valere deve essere efficace. Questo significa che non si può parlare di Costituzione senza parlare di governo, di comando, di istanze decisionali che discriminano dando luogo a conflitto e dissenso. In termini tradizionali tutto questo vuol dire Stato e statualità. Parlare di una Costituzione europea «senza Stato» (o più pudicamente «oltre lo Stato») non è serio.

Di fronte a questa problematica appaiono insufficienti le filosofie sul costituzionalismo politico che si limitano a insistere sull'obiettivo della promozione dello status (o della Wertidee) della cittadinanza e la creazione della «buona società» ${ }^{33}$. Ben venga il superamento del contrasto tra vecchio e nuovo costituzionalismo, se quest'ultimo è concepito come «modellabilità istituzionale e giuridica della politica» (Ulrich K. Preuss). $\mathrm{Ma}$ questa operazione di «razionalizzazione e civilizzazione del politico» tramite la Costituzione, con pretese normative sempre più esplicite $^{34}$ corre grossi rischi. Per suo tramite infatti fa capolino

32 «Una Costituzione europea aperta è condizionata dal crescere oltre lo Stato nazionale europeo. Il fondamento giuridico per questo è la decisione dello Stato costituzionale per una statualità aperta in questa senso. Come possa o debba realizzarsi è oggi oscuro» (Beutler 1996, 60).

33 Il «costituzionalismo politico» ha come compito «incoraggiare e rafforzare le abitudini di cooperazione e la cultura del compromesso ragionato che sta alla base della risoluzione dei conflitti sociali dentro ad un quadro democratico (...). L'enfasi sulla dimensione politica della Costituzione e sull'importanza del metodo democratico come base per la legittimazione delle istituzioni e l'identificazione dei cittadini non deve essere inteso come un appello populistico alla costituzione della "maggioranza" o una negazione dei principi della separazione dei poteri. Al contrario». Così Bellamy e Castiglione (1996b, 66-67).

34 «Il concetto di una Costituzione basata sugli elementi normativi della citizen- 
il sempre verde utopismo democratico che attraverso la Costituzione (nuova o modificata) ripropone l'utopia della rivoluzione democratica radicale o quanto meno il primato di una società civile trasfigurata e idealizzata oltre misura. Di conseguenza rischia di attribuire alla giurisdizione costituzionale una funzione pedagogico-costruttivistica che in altre congiunture storiche aveva assegnato ad altre istanze politiche (al partito politico). Nella realtà poi questo atteggiamento si trova nella contraddizione di approvare in linea di principio la funzione giurisdizionale della Corte ( Custode della Costituzione di una società civile») nel momento stesso in cui la critica per la sua scarsa «discorsività». Senza contare che deve scontrarsi con una lunga serie di sentenze che non approva - come dimostra il caso della sinistra tedesca.

Ma si profila un altro pericolo: quello del «populismo democratico», quando l'appello al popolo depositario del potere costituente viene piegato contingentemente alla tattica politica o alla filosofia politica di una parte. Il popolo elettore-sovrano viene contrapposto bic et nunc al sistema politico-costituzionale esistente (come in parte è avvenuto con la protesta del «popolo bavarese» nell'estate 1995) con la pretesa di relativizzare ogni istanza di garanzia generale. Si genera così una distorsione di quella che dovrebbe essere la convergenza ideale «tra la Costituzione-garanzia, vale a dire intesa come suprema forma di tutela dei diritti e delle sfere individuali di libertà, e la Costituzioneindirizzo come documento che dà l'indirizzo fondamentale da seguire per la realizzazione dei valori costituzionali»»35. Nel populismo democratico infatti la Costituzione-indirizzo è resa funzionale alla promozione di alcuni «valori costituzionali» intesi partigianamente come favorevoli ad una parte politica piuttosto che ad un'altra. Come non mai, in questo caso, il ruolo della Corte costituzionale diventa «politico» e motivo di conflitto ${ }^{36}$.

ship è chiaramente orientato a collegare il vincolo di una unità genuinamente politica con la discorsività di una associazione volontaria (...). Soltanto il processo di un atto costituente comune produce quello che presuppone la Costituzione: citizens che si riconoscono reciprocamente uguali». Così Preuss (1994a, 28- 29).

35 Così Portinaro $(1996,31)$. Si veda anche Dogliani (1996).

36 Il caso del «crocefisso» nelle scuole della Baviera ha mostrato alcuni tratti di questo potenziale populismo democratico. In Italia esso ha fatto e fa capolino in talune forme di pressione per riforme costituzionali palesemente funzionali alla tattica politica di questo o quel partito. Nel caso italiano, tuttavia, la Corte è stata risparmiata sinora dal prendere posizione. 
In realtà la sintesi tra Costituzione-garanzia e Costituzioneindirizzo produce sempre un sovraccarico di impegno che si ripercuote sull'attività della Corte costituzionale, creando i problemi che conosciamo. Di fronte a questa situazione sono state individuate tre linee di lettura critica che sono nel contempo motivi di un possibile intervento: conservatorismo costituzionale, ipercostituzionalismo e decostituzionalizzazione (Portinaro 1996).

Questo è lo sfondo in cui va posto il tema della politica della Costituzione nei suoi elementi caratterizzanti, sopra ricordati, dello statuto delle libertà, del contratto politico, della forma di governo. La formula «democrazia costituzionale» lascia alle spalle molti dei contrasti tradizionali tra il principio del «costituzionalismo» e quello della «democrazia». Le questioni della giustizia costituzionale, le frizioni che nascono al confine tra le competenze del legislativo e le competenze del giudiziario non sono segno di una patologia politica né di una variante dell'anti-politica. Segnalano una forma del politico tipica appunto della democrazia costituzionale che è il nostro futuro, soprattutto se e quando andrà in porto la costruzione/costituzione europea. A fronte delle insicurezze della dottrina giuridica e della pratica politica, le Corti - in particolare la Corte europea - stanno tessendo in modo pragmatico e incrementale, attraverso decisioni, pronunciamenti ad boc e creazioni di procedure, una rete di vincoli che non sappiamo ancora se saranno sufficienti a «tenere insieme», a «costituire» davvero l'Europa. A tutt'oggi è difficile dire se questa impresa avrà successo.

\section{Riferimenti bibliografici}

Abromeit, H. (1995), Volkssouveränität, Parlamentssouveränität, Verfassungssouveränität: Drei Realmodelle der Legitimation staatlichen Handelns, in «Politische Vierteljahresschrift», 36, 1, pp. 49-66.

Bellamy, R. e D. Castiglione (a cura di) (1996a), Constitutionalism in Transformation: European and Theoretical Perspectives, in «Political Studies», XLIV, Special Issue.

- (1996b), Costituzionalismo e democrazia in prospettiva europea, in «Teoria politica», 3, pp. 47-70.

Beutler, B. (1996), Auf dem Weg zu einer europäischen Verfassung, in «Kritische Justiz», 1, pp. 53-64.

Beyme von, K. (1988), The Genesis of Constitutional Review in Parliamentary Systems, in Landfried (1988b), pp. 21-38. 
Böckenförde, E.W. (1976), Die politische Funktion wirtschaftlich-sozialer Verbände und Interessenträger in der sozial-staatlichen Demokratie, ora in Böckenförde (1991), pp. 406-439.

- (1981), Geschichtliche Entwicklung und Bedeutungswandel der Verfassung, ora in Böckenförde (1991), pp. 29-42.

- (1986), Die verfassunggebende Gewalt des Volkes - Ein Grenzbegriff des Verfassungsrechts, ora in Böckenförde (1991), pp. 90-112.

- (1991), Staat, Verfassung, Demokratie, Frankfurt a.M., Suhrkamp.

- (1996a) Dem Bundesverfassungsgericht drobt der Kollaps, in «Frankfurter Allgemeine Zeitung», 24 maggio.

- (1996b), Il potere costituente del popolo, in Zagrebelsky, Portinaro e Luther (1996), pp. 231-252.

Denninger, E. (1995), Der einzelne und das allgemeine Gesetz, in «Kritische Justiz», 28, 4, pp. 425-450.

- (1996), Menschenrechte und Staatsaufgabe - ein «europäisches» Thema, relazione tenuta al Simposio Das Endziel der europäischen Integration. Von den Nachkriegsordnungen zu einem europäischen Konstitutionalismus?, Europäische Akademie Berlin, 2-4 maggio.

Dogliani, M. (1996), Potere costituente e revisione costituzionale nella lotta per la costituzione, in Zagrebelsky, Portinaro e Luther (1996), pp. 253-289.

Due, O., M. Lutter e J. Schwarze (a cura di) (1995), Festschrift für Ulrich Everling, Baden Baden, Nomos.

Elkin, S.L. e K.E. Soltan (a cura di) (1993), A new Costitutionalism. Designing Political Institutions for a Good Society, Chicago-London.

Frankenberg, G. (a cura di) (1994), Auf der Suche nach der gerechten Gesellschaft, Frankfurt a.M., Fischer.

- (1996), Hüter der Verfassung einer Zivilgesellschaft, in «Kritische Justiz», 1, pp. 1-14.

Frowein, J.A. (1994), Das Maastricht-Urteil und die Grenzen der Verfassungsgerichtsbarkeit, in «Zeitschrift für ausländisches und öffentliches Recht und Völkerrecht», 54, pp. 1-16.

Grimm, D. (1994), Braucht Europa eine Verfassung?, München, Fondazione Carl Friedrich von Siemens.

Guarnieri, C. e P. Pederzoli (1997), La democrazia giudiziaria, Bologna, Il Mulino.

Häberle, P. (1993), Le libertà fondamentali nello Stato costituzionale, Roma, La Nuova Italia Scientifica.

- (1994), I diritti fondamentali nelle società pluraliste e la Costituzione del pluralismo, in Luciani (1994), pp. 93-173.

Isensee, J. (1989), Die Verfassung als Vaterland. Zur Staatsverdrängung der Deutschen, in Mohler (1989), pp. 11-36.

- (1995), Integrationsziel Europastaat? in Due, Lutter e Schwarze (1995), pp. 567-592.

Isensee, J. e P. Kirchhof (a cura di) (1995), Die Einbeit Deutschlands, 
Entwicklung und Grundlagen, VIII volume dello Handbuch des Staatsrechts der Bundesrepublik Deutschland, Heidelberg, Müller.

Landfried, C. (1988a), Constitutional Review and Legislation in the Federal Republic of Germany, in Landfried (1988b), pp. 147-168.

- (a cura di) (1988b), Constitutional Review and Legislation. An International Comparison, Baden Baden, Nomos.

Luciani, M. (a cura di) (1994), La democrazia alla fine del secolo, Roma-Bari, Laterza.

Matteucci, N. (1995), Lo Stato moderno, Bologna, Il Mulino.

Maus, I. (1992) Zur Aufklärung der Demokratietheorie, Frankfurt a.M., Suhrkamp.

- (1994), Volkssouveränität versus Konstitutionalismus, in Frankenberg (1994), pp. 74-83.

Mohler, A. (a cura di) (1989), Wirklichkeit als Tabu, München, Oldenbourg.

Pernice, I. (1995), Deutschland in der Europäischen Union, in Isensee e Kirchhof (1995), pp. 225-280.

Pizzetti, F. (1996), Federalismo, regionalismo e riforma dello Stato, Torino, Giappichelli.

Portinaro, P.P. (1996), Il grande legislatore e il custode della Costituzione in Zagrebelsky, Portinaro e Luther (1996), pp. 5-34.

Preuss, U.K. (1994a), Der Begriff der Verfassung und ibre Beziebung zur Politik, in Preuss (1994b), pp. 7-33.

- (a cura di) (1994b), Zum Begriff der Verfassung. Die Ordnung des Politischen, Frankfurt, Fischer.

Rusconi, G.E. (1993), Se cessiamo di essere una nazione, Bologna, Il Mulino.

- (1997), Patria e repubblica, Bologna, il Mulino.

Sartori, G. (1987), Costituzione in Elementi di teoria politica, Bologna, Il Mulino.

Tomuschat, C. (1993), Die Europäische Union unter Aufsicht des Bundesverfassungsgerichts, in «Europäische Grundrechte-Zeitschrift», 3.

- (1996), Das Endziel der europäischen Integration: Staat, internationales Zusammenschluss oder «monstro simile»?, Relazione tenuta al Simposio Das Endziel der europäischen Integration. Von den $\mathrm{Na}$ chkriegsordnungen zu einem europäischen Konstitutionalismus?, Europäische Akademie Berlin, 2-4 maggio 1996.

Weber, M. (1961), Economia e società, vol. 1, Milano, Comunità.

Weiler, J.H.H. (1995), The State «über alles». Demos, Telos und the German Maastricht Decision, in Due, Lutter e Schwarze (1995), pp. 1651-1688.

Zagrebelsky, G. (1992), Il diritto mite, Torino, Einaudi.

Zagrebelsky, G., P.P. Portinaro e J. Luther (a cura di) (1996), Il futuro della Costituzione, Torino, Einaudi. 